\title{
Las fitocenosis de Cosentinia vellea en el sector biogeográfico Lusitano Duriense (España-Portugal)
}

\author{
Francisco Amich y Sonia Bernardos ${ }^{1}$
}

Resumen: Amich, F. \& Bernardos, S. 2013. Las fitocenosis de Cosentinia vellea en el sector biogeográfico Lusitano Duriense (España-Portugal). Bot. Complut. 37: 93-98.

Estudiamos en el presente trabajo el comportamiento fitosociológico de las comunidades vegetales caracterizadas por la presencia de $C_{0}$ sentinia vellea en el sector biogeográfico Lusitano Duriense. Tras un análisis detallado de las mismas, y su comparación con fitocenosis afines, consideramos que deben ser incluidas en la asociación Cheilantho maderensis-Cosentinietum velleae. Asimismo, analizamos y discutimos su encuadre en unidades superiores.

Palabras clave: comunidades de Cosentinia, río Duero, Península Ibérica, Lusitano-Duriense.

Abstract: Amich, F. \& Bernardos, S. 2013. Cosentinia vellea communities in the Lusitan Duriensean biogeographical sector (Spain-Portugal). Bot. Complut. 37: 93-98.

We study in this paper the phytosociological behavior of plant communities characterized by the presence of Cosentinia vellea in the Lusitan-Duriensean biogeographical sector. After a detailed analysis of the same, and its comparison with related phytocoenoses, we believe should be included in the association Cheilantho maderensis-Cosentinietum velleae. Finally we analyze and discuss the framework in higher units.

Key words: Cosentinia communities, Duero River, Iberian Peninsula, Lusitan-Duriensean.

\section{INTRODUCCIÓN}

Cosentinia vellea es un pteridófito que, en la Península Ibérica, se distribuye fundamentalmente por las zonas mediterráneas (Muñoz Garmendia 1986), donde no se trata de una planta frecuente. En el cuadrante noroccidental ibérico es asimismo un taxon bastante raro, y únicamente se conoce de las provincias de Salamanca (España) y Trás-0s-Montes (Portugal) (Amich \& Sánchez Rodríguez 1986, Muñoz Garmendia 1986).

Las comunidades en que se integra han constituido siempre un tema de debate y su posición sintaxonómica en los territorios estudiados, y también en otras zonas peninsulares, se encuentra aún sin resolver de forma definitiva. Ladero (1970), en su trabajo sobre la provincia de Cáceres, considera que Cosentinia vellea se integra en comunidades relícticas que denomina Cheilantheto pteridioido-catanensis. Posteriormente Amich \& Sánchez Rodríguez (1986) y Santos et al. (1988) denominan a esta comunidad Cheilantho maderensis-Cosentinietum velleae, denominación que se corresponde con la correcta nomenclatura actual de los taxones que la integran. Con posterioridad, Pérez-Carro et al. (1989) validan el nombre de la asociación descrita por Ladero (1970), aunque el inventario tipo de la misma no se corresponde con los territorios occidentales ibéricos donde se describió, sino al Cabo de Gata (Almería).

Es nuestro objetivo en este trabajo definir y delimitar las fitocenosis en que vive Cosentinia vellea en el cuadrante noroccidental ibérico, así como analizar las relaciones y encuadre en unidades superiores, y su comparación con otras asociaciones próximas.

\section{MATERIALES Y MÉTODOS}

Área de estudio. El area estudiada comprende una serie de territorios situados en el sector biogeográfico Lusitano Duriense (subprovincia Carpetano Leonesa, provincia Mediterránea Ibérica Occidental, Rivas-Martínez et al. 2002). Estos territo-

\footnotetext{
${ }^{1}$ Departamento de Botánica, Facultad de Biología, Universidad de Salamanca, E-37007 Salamanca, España. amich@usal.es, bernardos@usal.es

Recibido: 31 enero 2013. Aceptado: 21 febrero 2013.
} 
rios se extienden a lo largo de las riberas del río Duero y alguno de sus principales afluentes (ríos Águeda, Huebra, Uces, Côa y Sabor) (Fig. 1). La localización geográfica y biogeográfica de esta área de estudio, en los límites entre el mundo mediterráneo y el eurosiberiano, hace que sea muy rica y compleja florísticamente, con numerosas disyunciones y especies endémicas. Así, está muy bien caracterizada por táxones endémicos o subendémicos lusitano durienses, tales como: Allium schmitzii, Anarrhinum duriminium, Antirrhinum lopesianum, Holcus setiglumis subsp. duriensis, Scrophularia valdesii, Silene boryi subsp. duriensis y Trigonella polyceratia subsp. amandiana, o por taxones cuya distribución principal está centrada en estas áreas: Delphinium fissum subsp. sordidum, Isatis platyloba y Linaria intricata (= L. coutinhoi). Desde el punto de vista de la vegetación, estos territorios están caracterizados por mesobosques climatófilos de la asociación Physospermo cornubiensis-Quercetum suberis Rivas-Martínez 1987 y por microbosques y mesobosques climatófilos y edafoxerófilos de la sociación Junipero lagunae-Quercetum suberis Rivas-Martínez, Aguiar, Cantó \& Ladero 2002. Las principales características litológicas y bioclimáticas del area de estudio fueron descritas en detalle en Amich et al. (2004) y Bernardos et al. (2004).

Este estudio comprende un total de 27 especies y 13 inven- tarios, y ha sido realizado siguiendo el método de Braun-Blanquet (1979). La nomenclatura sintaxonómica sigue la propuesta por Rivas-Martínez et al. (2002), excepto para el sintaxon Notholaeno marantae-Cheilanthetalia maderensis Sáenz \& RivasMartínez in Itinera Geobot. 18: 218 (2011). En la sinopsis sintaxonómica de los sintaxones citados en el texto, seguimos asímismo la numeración y orden de Rivas-Martínez et al. (2002).

La nomenclatura usada para las especies anteriormente indicadas, así como todas aquellas de interés sintaxonómico es la de Flora Europaea (Tutin et al. 1964-1980) y Flora Iberica (Castroviejo 1986-2012), excepto para Silene boryi subsp. duriensis (Samp.) Coutinho in Fl. Portugal: 220 (1913) y para Trigonella polyceratia subsp. amandiana (Samp.) Amich \& J. Sánchez in Stud. Bot. Univ. Salamanca 2: 129 (1983).

\section{RESULTADOS Y DISCUSIÓN}

Hemos realizado un total de 13 inventarios, tanto en aquellas localidades en que había sido citada Cosentinia vellea, como en otras donde no está presente, que aparecen recogidos en la Tabla 1 .

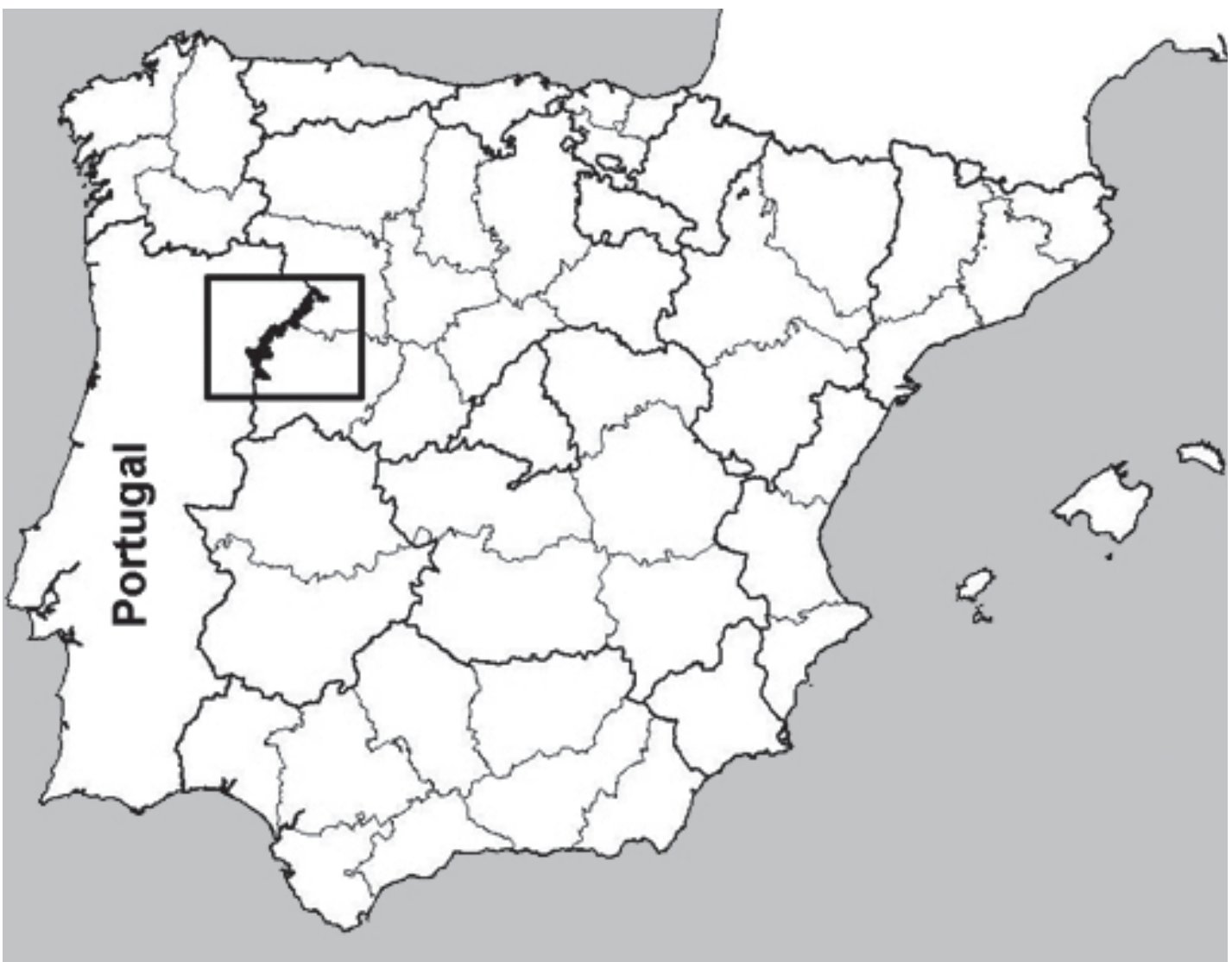

Figura 1- Área de estudio. 
Tabla 1

Cheilantho maderensis-Cosentinietum velleae (inventarios números 1-10)

(Notholaeno marantae-Cheilanthetalia maderensis, Asplenietea trichomanis)

(Comunidades sin Cosentinia vellaea, y con Cheilanthes maderensis o Cheilanthes tinaei (inventarios números 11-13)

\begin{tabular}{lrrrrrrrrrrrrr}
\hline Número de orden & 1 & 2 & 3 & 4 & 5 & 6 & 7 & 8 & 9 & 10 & 11 & 12 & 13 \\
Area $\left(\mathrm{m}^{2}\right)$ & 10 & 10 & 4 & 5 & 5 & 5 & 4 & 1,5 & 2 & 3 & 2 & 5 & 5 \\
Cobertura (\%) & 35 & 25 & 65 & 55 & 45 & 60 & 45 & 20 & 30 & 20 & 40 & 20 & 30 \\
Inclinación (\%) & 65 & 65 & 85 & 80 & 80 & 85 & 90 & 60 & 60 & 90 & 70 & 60 & 75 \\
Altitud (1=10 m) & 18 & 16 & 17 & 17 & 17 & 16 & 16 & 19 & 17 & 29 & 42 & 15 & 22 \\
Exposición & $\mathrm{W}$ & $\mathrm{SW}$ & $\mathrm{S}$ & $\mathrm{S}$ & $\mathrm{S}$ & $\mathrm{S}$ & $\mathrm{S}$ & $\mathrm{W}$ & $\mathrm{W}$ & $\mathrm{S}$ & $\mathrm{S}$ & $\mathrm{W}$ & $\mathrm{S}$ \\
\hline
\end{tabular}

Características de asociación, alianza, orden y clase

$\begin{array}{llllllllllllll}\text { Cosentinia velleae } & 2 & 4 & 3 & 3 & 3 & 4 & 4 & 2 & 2 & 1 & - & - & - \\ \text { Phagnalon saxatile } & 3 & 1 & 2 & 2 & 3 & 3 & 4 & 1 & 1 & 2 & 1 & 1 & - \\ \text { Rumex induratus } & 4 & 1 & 1 & 1 & 2 & 3 & - & - & 1 & 1 & 1 & 1 & - \\ \text { Anarrhinum bellidifolium } & 1 & + & - & 1 & - & 1 & 1 & - & 1 & 1 & - & - & - \\ \text { Antirrhinum graniticum } & + & + & - & 1 & - & - & - & - & - & 1 & - & - & - \\ \text { Dianthus lusitanus } & - & - & - & 1 & - & 1 & - & - & - & - & 1 & - & - \\ \text { Sedum hirsutum } & - & + & - & - & - & - & - & - & - & - & 1 & - & 1 \\ \text { Arisarum simorrhinum } & 2 & 1 & - & - & - & - & - & - & - & - & - & - & -\end{array}$

Especies acompañantes

$\begin{array}{llllllllllllll}\text { Sedum album } & 3 & 1 & 2 & 1 & - & 1 & - & 1 & - & 2 & 1 & 1 & 1 \\ \text { Umbilicus rupestris } & 2 & 1 & 1 & - & - & - & - & 1 & 1 & 1 & 1 & - & 1 \\ \text { Sedum caespitosum } & - & - & - & - & - & - & - & 1 & 1 & - & - & 1 & - \\ \text { Cheilanthes tinaei } & - & - & - & - & - & - & - & - & - & 2 & - & 3 & 2 \\ \text { Cheilanthes hispanica } & 1 & 1 & 1 & - & - & - & - & - & - & - & - & - & - \\ \text { Cheilanthes maderensis } & - & - & - & - & - & - & - & 2 & - & - & 3 & - & - \\ \text { Arrhenaterum elatius } & - & - & + & + & + & + & + & - & - & - & - & - & \\ \text { Silene inaperta } & + & + & - & 1 & - & - & - & - & - & - & 1 & - & - \\ \text { Brachypodium dystachium } & - & - & - & 1 & - & - & - & - & 1 & - & - & - & - \\ \text { Sanguisorba minor } & 1 & 1 & - & - & - & - & - & - & - & 1 & - & - & - \\ \text { Ceterach officinarum } & - & - & - & - & - & - & - & - & - & - & 1 & - & 1 \\ \text { Targionia hypophylla } & 1 & 1 & 1 & 1 & 1 & 1 & 1 & 1 & 1 & 1 & 1 & 1 & 1 \\ \text { Mannia androgyna } & 1 & 1 & 1 & 1 & 1 & 1 & 1 & 1 & 1 & - & - & 1 & 1 \\ \text { Reboulia hemisphaerica } & - & - & - & - & - & - & - & 1 & 1 & 1 & 1 & 1 & 1\end{array}$

Además: Crucianella angustifolia + en 1; Sedum arenarium 1 en 2; Andropogon hirtus 4 en 6; Oxymitra paleacea y Cladonia gr. fimbriata 1 en 8.

Localidades de los inventarios: 1: Portugal, Trás-os-Montes, Freixo, Barca d'Alva, riberas del río Duero, 29TPF7744, (4101' 57,2” N $-06^{0} 53$ ' 15,6”'W), 10.11.2004, Amich \& Bernardos. 2: Portugal, Trás-0s-Montes, Freixo, Barca d'Alva, riberas del río Duero, 29TPF7544, (41 02 ' 6" N - 06 54' 12" W), 10.11.2004, Amich \& Bernardos. 3: Portugal, Trás-os-Montes, Freixo, Barca d'Alva, riberas del río

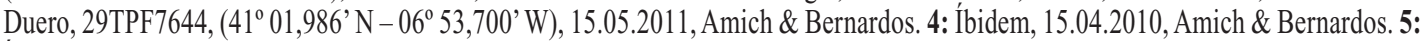
Íbidem, 15.04.2010, Amich \& Bernardos. 6: Portugal, Trás-os-Montes, Freixo, Barca d'Alva, riberas del río Duero, 29TPF7644, $\left(41^{\circ}\right.$ 02,016’ N - 06 53,845’ W), 05.05.2012, Amich \& Bernardos. 7: Portugal, Trás-os-Montes, Freixo, Barca d'Alva, riberas del río Duero, 29TPF7644, (41 02,086' N - 06 54,101’ W), 05.05.2012, Amich \& Bernardos. 8: Spain, Salamanca, La Fregeneda, Muelle de Ve-

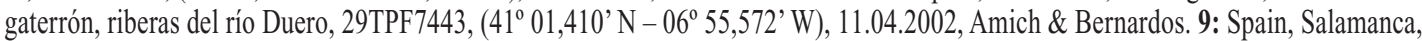
La Fregeneda, Muelle de Vegaterrón, riberas del río Duero, 29TPF7443, (41 ${ }^{\circ} 01,453^{\prime} \mathrm{N}$ - 06 55,671' W), 21.03.2007, Amich \& Bernardos. 10: Spain, Salamanca, Lumbrales, Puente de la Molinera, riberas del río Huebra, $29 \mathrm{TPF} 9044\left(41^{\circ} 01,816^{\prime} \mathrm{N}-06^{\circ} 43,875^{\prime} \mathrm{W}\right)$, 12.01.2013, Amich \& Bernardos. 11: Spain, between Lumbrales and Saucelle, km. 19, riberas del río Huebra, 29TPF8945, (41 02,266' N-06 44,581' W), 10.05.2008, Amich \& Bernardos. 12: Spain, Salamanca, La Fregeneda, Muelle de Vegaterrón, riberas del río Duero, 29TPF7443, $\left(41^{\circ} 01,635^{\prime} \mathrm{N}-06^{\circ} 55,764^{\prime} \mathrm{W}\right), 15.05 .2011$, Amich \& Bernardos. 13: Spain, Salamanca, Saucelle, Salto de Saucelle, riberas del río Duero, 29TPF8445 (41ํ02,420’ N - 06 48,394’ W), 12.01.2013, Amich \& Bernardos. 
La primera cuestión a tener en cuenta es la clase fitosociológica a la que asignar las comunidades estudiadas en este trabajo, dado que muchas de ellas, por su composición florística general (ver Tabla 1), y en concreto por la presencia de taxones como Cosentinia vellea y Cheilanthes maderensis, podrían relacionarse con las propias de la clase Asplenietea trichomanis, en concreto con las pertenecientes a la alianza Phagnalo-Cheilanthion maderensis (Notholaeno marantae-Cheilanthetalia maderensis), aunque algunas de las especies características de la misma (i.e. Cheilanthes maderensis) apenas están presentes en las comunidades estudiadas: se presenta junto con Cosentinia exclusivamente en el inventario $\mathrm{n}^{0} 8$, y está ausente en casi todas las localidades restantes.

Dentro de Asplenietea, también hemos analizado su posible pertenencia a la alianza Cheilanthion hispanicae (Androsacetalia vandellii), pero sin embargo faltan 0 están muy escasamente representadas diversas especies características de dicha alianza, tales como Cheilanthes hispanica, C. tinaei, Asplenium billotii o Sedum brevifolium.

El análisis de la topografia (fisuras de poca inclinación e incluso derrubios y taludes terrosos, con apenas táxones casmofíticos) y fisionomía de estas fitocenosis (integradas fundamentalmente por caméfitos y hemicriptófitos) podría llevar a encuadrarlas dentro de la clase Phagnalo-Rumicetea, en concreto en la alianza RumiciDianthion, alianza extraordinariamente diversificada y rica en estos territorios lusitano durienses, con un total de 4 asociaciones (Bernardos et al. 2004). Asimismo son frecuentes en estas comunidades taxones característicos de Phagnalo-Rumicetea, tales como Rumex induratus, Dianthus lusitanus, Antirrhinum graniticum, etc. Sin embargo, no es infrecuente que tales especies aparezcan como compañeras en diversos sintaxones de Asplenietea trichomanis. Por otro lado, las especies de Cheilanthes son muy raras en Phagnalo-Rumicetea, y bastante más comunes en Asplenietea.

Por todo lo anteriormente indicado, hemos de admitir que las comunidades estudiadas, a pesar de la poca presencia en las mismas de Cheilanthes maderensis, deben ser incluidas en la alianza Phagnalo saxatile-Cheilanthion maderensis (Notholaeno marantae-Cheilanthetalia maderensis), que agrupa las asociaciones casmofíticas pteridofíticas mediterráneas, infra-supramediterráneas semiárido-húmedas (Rivas-Martínez 2011), y representaría una comunidad finícola de dicha alianza.

En cuanto a la adscripción de estas fitocenosis lusi- tano durienses a una asociación concreta, en nuestra opinión la asociación Cheilantho maderensis-Cosentinietum velleae, no se corresponde exactamente en su concepción actual (Pérez-Carro et al. 1989), con la indicada por Ladero (1970) en su descripción original para los territorios toledano taganos. Sin embargo, nuestros inventarios números 1-10 de la Tabla 1 no se desvían de manera sustancial de los publicados por Pérez-Carro et al. (1989, Tabla 6), por lo que no parecen existir criterios razonables para no incluir nuestras comunidades en este mismo sintaxon.

Otro aspecto a tener en cuenta es si nuestras comunidades lusitano durienses podrían considerarse una raza geográfica particular de Cheilantho-Cosentinietum. Aunque podríamos considerar algunos taxones singulares como diferenciales, por ejemplo Antirrhinum graniticum y Dianthus lusitanus, al alcanzar ambos diversos territorios del sur y levante ibéricos, creemos que no serían buenos diferenciales desde el punto de vista biogeográfico. Tal vez la búsqueda y estudio de nuevas poblaciones y comunidades en las que esté presente Cosentinia vellea nos permita profundizar y resolver algunos de los aspectos pendientes.

Desde un punto de vista biogeográfico la alianza Phagnalo-Cheilanthion maderensis alcanza su óptimo en los territorios mediterráneos del levante y sur ibéricos y llega, de manera muy puntual y finícola, hasta algunos territorios carpetanos, a través de asociaciones basales de la alianza, como Notholaenetum marantae (Aguiar 2001, Rivas-Martínez et al. 2001, 2002). Por su parte, Cheilantho maderensis-Cosentinietum velleae quedaría restringida fundamentalmente a las provincias Bética y Murciano-Almeriense (Pérez-Carro et al. 1989), y alcanzaría puntos muy aislados y concretos de lo toledano tagano y lo lusitano duriense.

Descripción de Cheilantho maderensis-Cosentinietum velleae en los territorios Lusitano Durienses. Esta comunidad incluye fundamentalmente caméfitos y hemicriptófitos que viven preferentemente sobre suelos rocosos y que alfombran las grietas, fisuras y pequeños canchales con rocas sueltas, ácidas, principalmente granitos, gneises y cuarcitas.

En el sector biogeográfico Lusitano Duriense se presenta entre los 150 y $425 \mathrm{~m}$, en un termotipo mesomediterránero y un ombrotipo seco, y se encuadra en el seno de los bosques de la asociación Junipero lagunae-Quercetum suberis. En estos valles muy térmicos de la cuenca inferior del río Duero representaría el extremo del área de distribución de comunidades de Phagnalo-Cheilanthion, que aquí se difuminan y pier- 
den, 0 están muy poco representadas, algunas de sus especies características, mientras se enriquece en elementos endémicos ibéricos o ibero-norteafricanos de distribución preferentemente occidental, tales como Antirrhinum graniticum, Dianthus lusitanus, Rumex induratus y Sedum hirsutum, adaptándose de esta manera a las condiciones geomorfológicas, biogeográficas y bioclimáticas del territorio, y caracterizada florísticamente por el paleoelemento Cosentinia vellea, que alcanza en estas localidades su límite septentrional en la Peninsula Ibérica.

\section{CONCLUSIONES}

Tras nuestro estudio de las comunidades vegetales con Cosentinia vellea en el sector biogeográfico Lusitano Duriense, podemos concluir que en estos territorios la alianza mediterránea Phagnalo saxatile-Cheilanthion maderensis está representada por 2 asociaciones de las 7 que en ella se distinguen (Rivas-Martínez 2011), que alcanzan de manera finícola estos territorios: Notholaenetum marantae, caracterizada por la presencia de la reliquia paleotropical Notholaena marantae subsp. marantae, y endémica de las rocas ultrabásicas de NE de Trás-os-Montes (Portugal), y Cheilantho maderensis-Cosentinietum velleae.

\section{SINOPSIS SINTAXONÓMICA DE LOS SINTAXONES MENCIONADOS EN EL TEXTO}

27. Asplenietea trichomanis (Br.-Bl. in Meier \& Br.-Bl. 1934) Oberdorfer 1977

27b. Androsacetalia vandellii Br.-Bl. in Meier \& Br.-Bl. 1934

27.8. Cheilanthion hispanicae Rivas Goday 1955 em. Sáenz \& Rivas-Martínez 1979

27e. Notholaeno marantae-Cheilanthetalia maderensis Sáenz \& Rivas-Martínez 2011

27.18. Phagnalo saxatilis-Cheilanthion maderensis Loisel 1970 corr. F. J. Pérez, T. E. Díaz, P. Fernández \& Salvo 1989

27.18.5. Cheilantho maderensis-Cosentinietum velleae Ladero ex F. J. Pérez, T. E. Díaz, P. Fernández \& Salvo 1989

27.18.6. Notholaenetum marantae (P. Silva 1970) Capelo, Aguiar \& Gomes Pedro 1996

32. Phagnalo-Rumicetea indurati (Rivas Goday \& Esteve 1972) Rivas-Martínez, Izco \& Costa 1973

32a. Phagnalo saxatilis-Rumicetalia indurati Rivas Goday \& Esteve 1972

32.3. Rumici indurati-Dianthion lusitani Rivas-Martínez, Izco \& Costa 1973 ex Fuente 1986

\section{AGRADECIMIENTOS}

Agrademos al Dr. Tomás E. Díaz González sus valiosos comentarios y sugerencias, que han enriquecido la calidad y claridad del manuscrito original.

\section{BIBLIOGRAFÍA}

Aguiar, C. 2001. Flora e vegetaçao da serra de Nogueira e do Parque Natural de Montesinho. Unpublised PhD Thesis, Universidade Técnica de Lisboa.

Amich, F. \& SÁnchez-RodrígueZ, J. A. 1986. Acerca de la presencia de Cosentinia vellea (Aiton) Tod. en la provincia de Salamanca. Anales Jard. Bot. Madrid 42: 530.

Amich, F.; Bernardos, S.; Aguiar, C.; Fernández-Diez, F. \& CresPí, A. L. 2004. Taxonomic composition and ecological characteristics of the endemic flora of the lower Duero Basin (Iberian Peninsula). Acta Bot. Gallica 151: 341-352.

Bernardos, S.; Crespí, A. L.; Aguiar, C.; Fernández.-Diez, F. J. \& Amich, F. 2004. The plant communities of the Rumici indurati-Dianthion lusitani alliance in the Lusitan Duriensean biogeographical sector (NE Portugal and CW Spain). Acta Bot. Gallica 151: 147-164.

Braun-Blanquet, J. 1979. Fitosociología. Bases para el estudio de las comunidades vegetales. Blume, Madrid.

Castroviejo, S. (Coord. gen.). 1986-2012. Flora Iberica, 1-8, 10-15, 17-18, 21. Real Jardín Botánico, Madrid.

Ladero, M. 1970. Contribución al estudio de la flora y vegetación de las comarcas de la Jara, serranía de Ibor y Guadalupe-Villuercas, en la Oretana Central. Tesis Doctoral inéd. Facultad de Farmacia, Universidad Complutense, Madrid.

MuÑoz Garmendia, F. 1986. Cosentinia Tod. En: S. Castroviejo (Coord. gen.), Flora Iberica, 1: 65. Real Jardín Botánico, Madrid.

Pérez-Carro, F. J.; Díaz-González, T. E.; Fernández-AreceS, M. P. \& Salvo, E. 1989. Contribución al estudio de las comunidades rupícolas de la Cheilanthetalia marantomaderensis y Androsacetalia vandellii en la Península Ibérica. Acta Bot. Malac. 14: 171-191.

Rivas-MartíneZ, S. 2011. Mapa de series, geoseries y geopermaseries de vegetación de España. Itinera Geobot. 18: 5-800.

Rivas-Martínez, S.; Fernández-GonzÁlez, F.; Loidi, J.; Lous̃̃, M. \& PENAS, A. 2001. Syntaxonomical checklist of vascular plant communities of Spain and Portugal to association level. Itin. Geobot. 14: 5-341.

Rivas-Martínez, S.; Díaz, T. E.; Fernández-González, F.; 
Izco, J.; LoIdI, J.; LousÃ, M. \& PenAS, A. 2002. Vascular plant communities of Spain and Portugal. Itin. Geobot. 15: 5-922.

SANTOS, M. T.; LADERO, M. \& Amor, A. 1988. Vegetación de las intercalaciones básicas de la provincia de Cáceres (Extremadura, España). Studia Bot. 7: 9-146.

Tutin T. G. \& AL. (Eds.) 1964-1980. Flora Europaea, 1-5. Cambridge University Press, Cambridge. 